\title{
Intracellular expression of engineered RNase $P$ ribozymes effectively blocks gene expression and replication of human cytomegalovirus
}

\author{
KIHOON KIM, SEAN UMAMOTO, PHONG TRANG, RONG HAI, and FENYONG LIU \\ Program in Infectious Diseases and Immunity, Program in Comparative Biochemistry, School of Public Health, University of California, \\ Berkeley, California 94720, USA
}

\begin{abstract}
A ribozyme (M1GS RNA) constructed from the catalytic RNA subunit of RNase P from Escherichia coli was used to target the overlapping region of two human cytomegalovirus (HCMV) mRNAs, which encode for the viral essential protease (PR) and capsid assembly proteins (AP), respectively. The results show a reduction of $>80 \%$ in the expression levels of PR and AP and an inhibition of $\sim 2000$-fold of viral growth in cells that stably expressed the ribozyme. In comparison, $<10 \%$ reduction in the expression of the targets and viral growth was found in cells that either did not express the ribozyme or produced a "disabled" ribozyme carrying mutations that abolished its catalytic activity. Examination of replication of the virus in the ribozymeexpressing cells indicates that packaging of the viral genomic DNA into capsids is blocked, and suggests that the antiviral effects are because the ribozyme specifically inhibits the AP and PR expression and, consequently, abolishes viral capsid formation and growth. Our results show that RNase P ribozymes are highly effective in blocking HCMV growth by targeting the PR and AP mRNAs and demonstrate the feasibility to use these ribozymes in gene therapy for antiviral applications.
\end{abstract}

Keywords: Gene therapy; ribozyme; RNase P; cytomegalovirus; herpesvirus; gene targeting

\section{INTRODUCTION}

Human cytomegalovirus (HCMV) causes serious clinical manifestations in newborns and immunocompromised populations such as AIDS patients (Mocarski and Courcelle 2001; Pass 2001). In addition to diseases that are known to be the results of HCMV infection such as AIDS-associated CMV retinitis and birth defect-related metal disorders, there are growing numbers of clinically important complications that are believed to be associated with HCMV, including atherosclerosis (Pass 2001). HCMV is a member of herpesvirus family that includes many medically important viruses such as herpes simplex virus (HSV) 1 and 2 (causative agents of cold sore and genital herpes, respectively), Epstein-Barr virus (found in patients with Burkitt's lymphoma), and Kaposi's sarcoma-associated herpesviruses (KSHV; Kieff and Rickinson 2001; Mocarski and Courcelle

Reprint requests to: Fenyong Liu, Program in Infectious Diseases and Immunity, School of Public Health, 140 Warren Hall, University of California, Berkeley, CA 94720, USA; e-mail: liu_fy@uclink4.berkeley.edu; fax: (510) 643-9955.

Article and publication are at http://www.rnajournal.org/cgi/doi/ 10.1261/rna.5178404.
2001; Moore and Chang 2001; Roizman and Knipe 2001). Continued development of effective antiviral compounds and approaches is central for the treatment and prevention of infections by HCMV, as well as other herpesviruses.

RNA enzymes are being developed as promising genetargeting reagents to specifically cleave RNA sequences of choice (Sarver et al. 1990; Yu et al. 1993; Liu and Altman 1995; Lan et al. 1998; Guo et al. 2000). For example, both hammerhead and hairpin ribozymes have been shown to cleave viral mRNA sequences and inhibit viral replication in cells infected with human viruses, whereas a ribozyme derived from a group I intron has been used to repair mutant mRNAs in cells (Sarver et al. 1990; Yu et al. 1993; Lan et al. 1998; Wong-Staal et al. 1998; Rossi 2000). Thus, ribozymes can be used as a tool in both basic and clinical research, such as in studies of developmental processes and in antiviral gene therapy (Wong-Staal et al. 1998; Rossi 1999).

Ribonuclease P (RNase P) is a ribonucleoprotein complex responsible for the maturation of the $5^{\prime}$ termini of tRNAs (Frank and Pace 1998; Altman and Kirsebom 1999). In bacteria, the RNase P holoenzyme contains a catalytic RNA subunit (Guerrier-Takada et al. 1983) and a small basic protein subunit (e.g., M1 RNA and C5 protein in 
(A)

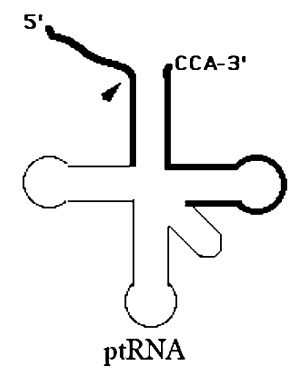

(B) ap40

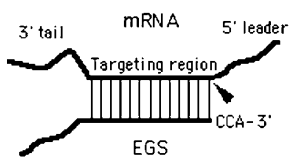

EGS:mRNA

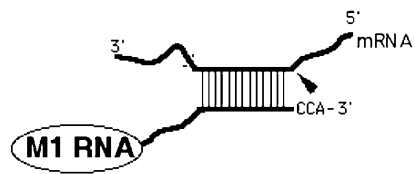

M1GS : mRNA

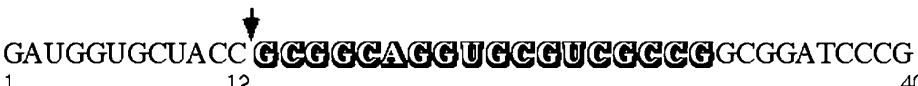

FIGURE 1. (A) Schematic representation of a natural substrate (ptRNA), a small model substrate (EGS:mRNA) for ribonuclease P and M1 RNA from Escherichia coli, and a complex formed between a M1GS RNA and its mRNA substrate. A part of the EGS:mRNA and M1GS:mRNA complexes resembles the acceptor stem and T-stem domains of a ptRNA (shown in bold). The site of cleavage by RNase P or M1 RNA is labeled with a filled arrow. $(B)$ Schematic representation of the substrate used in the study. The targeted sequences that bind to the guide sequences of the ribozymes are boxed.

Escherichia coli). One of the unique features of RNase P holoenzyme and its catalytic RNA is their ability to recognize the structures rather than the sequences of their substrates, which gives them the ability to hydrolyze different substrates. Thus, M1 ribozyme can cleave an mRNA substrate as long as the target sequence hybridizes with its complementary sequence (designated as external guide sequence or EGS) to form a complex resembling the portion of a tRNA molecule that includes the acceptor stem, the T-stem, the $3^{\prime}$ CCA sequence, and the $5^{\prime}$ leader sequence (Fig. 1A; Forster and Altman 1990). A sequence-specific ribozyme, M1GS RNA, can be constructed by covalently linking a guide sequence to the $3^{\prime}$ terminus of M1 RNA (Fig. 1A; Frank et al. 1994; Liu and Altman 1995). RNase P ribozymes have been constructed and used to inhibit the expression of both cellular genes and genes of HSV-1 and HCMV (Cobaleda and Sanchez-Garcia 2000; Trang et al. 2000a,b). Moreover, a reduction of 1000-fold in HSV-1 growth and a reduction of 150-fold in HCMV replication were observed in cells that expressed ribozymes derived from the wild-type RNase P ribozyme sequence (Trang et al. 2000a,b). Thus, M1GS ribozyme represents a novel class of gene-targeting agents. Targeted cleavage of mRNA by RNase P ribozyme provides a unique approach to inactivate any RNA of known sequence expressed in vivo.

In the present study, we constructed a M1GS ribozyme to target the overlapping region of the mRNAs coding for HCMV capsid assembly protein (AP) and protease (PR), and investigated the antiviral activity of the constructed ribozymes. The AP completely overlaps with and is within the 3' coding sequence of the viral PR (Welch et al. 1991). $\mathrm{AP}$ and PR are two of the most conserved proteins found among all herpesviruses (Liu and Roizman 1991; Welch et al. 1991). Genetic and biochemical studies of HSV-1 AP and

PR indicated that these two proteins are responsible for viral capsid assembly and are essential for HSV-1 replication (Preston et al. 1983; Gao et al. 1994; Matusick-Kumar et al. 1994). Therefore, the AP and the PR may serve as targets for novel drug development to combat all herpesviruses, including HCMV (Gibson et al. 1994).

The overlapping region of the $\mathrm{AP}$ and PR mRNAs is also an ideal target for M1GS targeting. This is because AP provides a stoichiometric function as the capsid scaffolding protein, whereas the proteolytic activity of PR is essential for capsid maturation (Preston et al. 1983; Gao et al. 1994; Gibson et al. 1994; Matusick-Kumar et al. 1994). Targeted cleavage of the common regions of AP and PR mRNA will achieve a greater antiviral effect, as it will shut down both the viral essential stoichiometric and enzymatic functions simultaneously. We showed that the constructed ribozyme cleaves the target mRNA sequence in vitro. Moreover, intracellular expression of the ribozyme using retroviral expression vectors leads to a significant inhibition of the expression of viral AP and PR. A reduction of 2000-fold in viral growth was observed in the ribozyme-expressing cells. Our study provides the direct evidence that RNase P ribozymes are highly effective in inhibiting HCMV gene expression and growth by targeting the mRNAs for AP and PR. These results also demonstrate the feasibility of developing highly effective RNase P ribozymes as a novel class of antiviral agents for gene therapy of infections caused by human viruses, including HCMV.

\section{RESULTS}

\section{Targeted cleavage of the AP mRNA sequence by M1GS ribozyme in vitro}

The mRNA (AP mRNA) encoding the HCMV capsid AP completely overlaps with and is within the PR mRNA, and these two mRNAs coterminate at the same $3^{\prime}$ polyadenenylation site (Welch et al. 1991). M1GS RNA-mediated cleavage at the overlapping region of the PR and AP mRNAs is expected to block the expression of both mRNAs simultaneously and should lead to a significant reduction of HCMV growth.

Most mRNA species inside cells are associated with proteins and are present in a highly organized and folded conformation. Therefore, it is important to choose a targeted region that is accessible to binding of ribozymes in order to achieve efficient cleavage. By using dimethyl sulfate (DMS), we used an in vivo mapping approach (Ares and Igel 1990; 
Liu and Altman 1995; Zaug and Cech 1995) to determine the accessibility of the region of the AP mRNA in HCMV-infected cells. A position 58 nucleotides downstream of the AP translational initiation codon (Chee et al. 1990), was chosen as the cleavage site for M1GS RNA. This site appears to be one of the regions most accessible to DMS modification and, presumably, to ribozyme binding. Moreover, its flanking sequence exhibits several features that need to be present in order to interact with an M1GS ribozyme to achieve efficient cleavage. The interactions of these sequence elements with the M1GS ribozyme are critical for recognition and cleavage by the enzyme. These features include the requirement for a pyrimidine and a guanosine to be the nucleotide $3^{\prime}$ and $5^{\prime}$ adjacent to the site of cleavage, respectively (Liu and Altman 1996).

Ribozyme M1-AP was constructed by covalently linking the $3^{\prime}$ terminus of M1 RNA with a guide sequence of 18 nucleotides that is complementary to the targeted AP mRNA sequence. The control ribozyme, C-AP, was also constructed in a similar way and included in the study. C-AP was derived from C102 RNA, a M1 mutant that contained several point mutations at the catalytic P4 domain and was at least $10^{4}$-fold less active than was M1 RNA in cleaving a pre-tRNA (Kim et al. 1997). The DNA sequences coding for the M1GS ribozymes were generated by PCR using the DNA sequences for M1 RNA and C102 RNA as the templates and primers that contained the sequences complementary to the targeted region of AP mRNA. These DNA sequences were under the control of the promoter for T7 RNA polymerase, and M1GS RNAs were synthesized in vitro from these DNA sequences by T7 RNA polymerase. A substrate, ap40, which contained the targeted AP mRNA sequence of 40 nucleotides, was used (Fig. 1B). In the absence of M1 RNAs (Fig. 2, lane 4), no cleavage of AP mRNA sequence was detected. Efficient cleavage of the substrate by M1-AP was observed (Fig. 2, lane 1). In contrast, cleavage by C-AP was barely detected (Fig. 2, lane 2).

Experiments with gel-shift assays were carried out to determine whether the differential cleavage efficiencies observed with M1-AP and C-AP were possibly due to their different binding affinities to the AP mRNA sequence. These results indicate that the binding affinity of C-AP to substrate ap40, measured as the dissociation constant $\left(\mathrm{K}_{\mathrm{d}}\right)$, is similar to that of M1-AP (data not shown). Because C-AP contains the same antisense guide sequence and similar affinity to ap40 as does M1-AP but is catalytically inactive, this ribozyme can be used as a control for the antisense effect in our experiments in cultured cells (see below).

\section{Efficient expression of the ribozymes in human cells}

The DNA sequences coding for M1-AP and C-AP were cloned into retroviral vector LXSN and placed under the control of the small nuclear U6 RNA promoter, which has previously been shown to express M1GS RNA and other

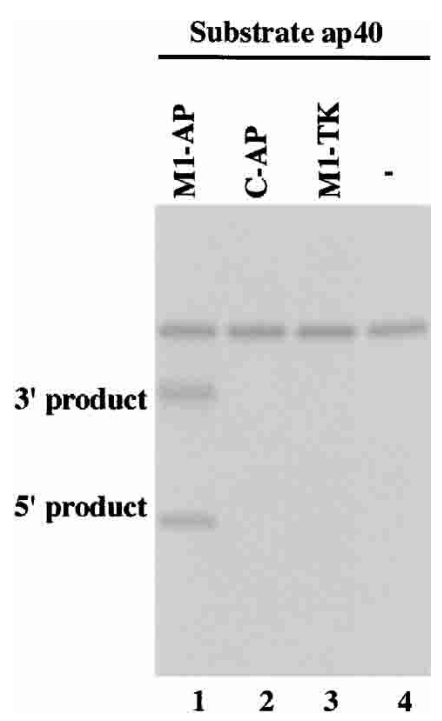

FIGURE 2. Cleavage of substrate ap40 by M1GS RNA. Substrate (20 $\mathrm{nM}$ ) was incubated alone (lane 4), with $5 \mathrm{nM}$ of M1-AP (lane 1), C-AP (lane 2), or M1-TK ribozyme (lane 3). Cleavage reactions were carried out for $30 \mathrm{~min}$ in buffer A $(50 \mathrm{mM}$ Tris. $\mathrm{HCl}$ at $\mathrm{pH} 7.5,100 \mathrm{mM}$ $\mathrm{NH}_{4} \mathrm{Cl}, 100 \mathrm{mM} \mathrm{MgCl} 2$ ) at $37^{\circ} \mathrm{C}$. Cleavage products were separated in $15 \%$ polyacrylamide gels containing $8 \mathrm{M}$ urea.

RNAs steadily (Das et al. 1988; Miller and Rosman 1989; Yuan et al. 1992; Liu and Altman 1995; Bertrand et al. 1997). This promoter is transcribed by RNA polymerase III, and its transcripts are highly expressed and primarily localized in the nucleus (Das et al. 1988; Yuan et al. 1992; Liu and Altman 1995; Bertrand et al. 1997). To construct cell lines that express M1GS ribozymes, amphotropic packaging cells (PA317; Miller and Rosman 1989) were transfected with LXSN-M1GS DNAs to produce retroviral vectors that contained the genes for M1GS RNA. Human U373MG cells were then infected with these vectors, and cells expressing the ribozymes were cloned.

An additional cell line, which expressed ribozyme M1-TK that targeted the mRNA for thymidine kinase (TK) of HSV-1 (Kilani et al. 2000), was also constructed. No cleavage of substrate ap40 by M1-TK was observed in vitro (Fig. 2 , lane 3$)$. We used this cell line to determine whether M1GS RNA with an incorrect guide sequence could target PR mRNA in tissue culture. The constructed lines and a control line in which cells were transfected with LXSN vector DNA alone were indistinguishable in terms of their growth and viability for up to 3 months (data not shown), suggesting that the expression of the ribozymes did not exhibit significant cytotoxicity.

We determined the level of M1GS RNA in each cell clone by using Northern analysis with a DNA probe that is complementary to M1 RNA. The M1GS RNAs were exclusively expressed in the nuclei as they were only detected in the nuclear but not the cytoplasmic RNA fractions (Fig. 3; data not shown). This is consistent with previous observations in our laboratory, as well as others, that the transcripts 


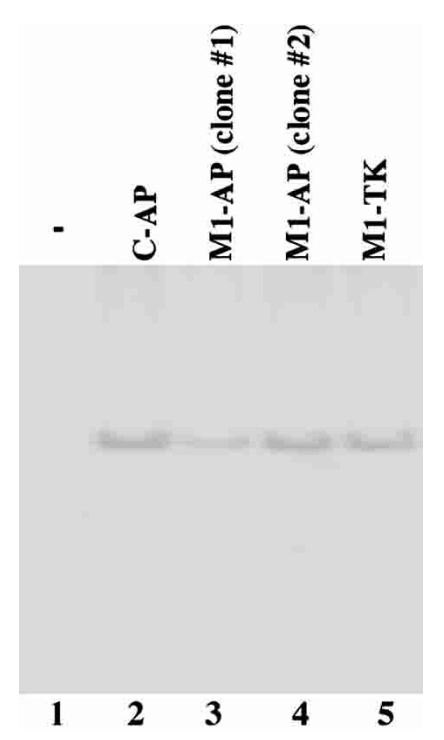

FIGURE 3. Expression of M1GS ribozymes in human cells, as detected by Northern analysis. Nuclear RNA fractions were isolated from parental U373MG cells (-, lane 1) or from a cell line that expressed C-AP (lane 2) and M1-TK (lane 5) and two different cell lines that expressed M1-AP (lanes 3,4). Equal amounts of each RNA sample (30 $\mu \mathrm{g})$ were separated on $2.5 \%$ agarose gels that contained formaldehyde, transferred to a nitrocellulose membrane, and hybridized to a $\left[{ }^{32} \mathrm{P}\right]$ radiolabeled probe that contained the DNA sequence coding for M1 RNA. The hybridized products corresponding to the full-length retroviral transcripts $(\sim 6 \mathrm{~kb})$, transcribed from the LTR promoter, are at the top of the gel and are not shown.

expressed by the U6 promoter are primarily localized in the nuclei (Yuan et al. 1992; Bertrand and Rossi 1995; Liu and Altman 1995; Good et al. 1997). The different levels of M1-AP expression between the two cloned cell lines (Fig. 3, cf. lanes 3 and 4) are presumably due to the incorporation of the LXSN-M1GS sequence into different locations of the host chromosome, and its expression is influenced by the flanking sequence at the insertion site. We only used the cell lines that expressed similar levels of these ribozymes for further studies in tissue culture.

\section{M1GS RNA-mediated inhibition of viral AP and PR expression}

To determine the efficacy of the ribozymes in inhibiting the expression of HCMV AP and PR, cells were infected with HCMV at a multiplicity of infection (MOI) of 0.05 to one. Total RNAs were isolated from the infected cells at 8-72 h postinfection. The expression levels of AP and PR mRNAs were determined by Northern analyses. The level of the 5-kb-long viral immediate-early (IE) transcript ( $5 \mathrm{~kb}$ RNA), the expression of which is not regulated by AP or PR under the assay conditions (Zhu et al. 1997), was used as an internal control for the quantitation of expression of AP and PR mRNAs (Fig. 4). A reduction of $\sim 85 \pm 9 \%$ and $88 \pm 9 \%$ (average of three experiments) in the expression level of PR and AP mRNA was observed in cells that expressed M1-AP, respectively (Table 1 ). In contrast, a reduction of $<10 \%$ in the expression level of these two mRNAs was observed in cells that expressed C-AP or M1-TK. These results suggest that the significant reduction of AP mRNA expression in cells that expressed M1-AP was due to the targeted cleavage by the ribozyme. The low level of inhibition found in cells that expressed C-AP was presumably due to an antisense effect because C-AP exhibits similar binding affinity to the target sequence as does M1-AP but is catalytically inactive.

The levels of AP and PR proteins in M1GS-expressing cells are expected to decline due to decreased levels of their mRNAs. Proteins were isolated from cells at $24-72 \mathrm{~h}$ postinfection, separated in SDS-polyacrylamide gels, and transferred to identical membranes. Two of these membranes were stained with an anti-PR and anti-AP antibody, respectively (Fig. 5B,C). Another membrane was stained with a monoclonal antibody against human actin (anti-actin; Fig. $5 \mathrm{~A})$. The latter serves as an internal control for the quantitation of AP and PR protein expression. The expression of $\mathrm{AP}, \mathrm{PR}$, and actin was quantitated by using a chemiluminescent substrate for the antibody staining (Fig. 5). The results of three independent experiments are summarized in Table 1: A reduction of $\sim 82 \%$ and $80 \%$ in the level of AP and PR proteins, respectively, was observed in cells that expressed M1-AP RNA. In contrast, a reduction of $<10 \%$ was found in cells that expressed C-AP or M1-TK RNAs. The low level of reduction in the expression level of AP and PR proteins observed in cells that expressed C-AP was probably due to the antisense effect of the guide sequence.

\section{Effective reduction of HCMV growth by M1GS targeting against AP and PR mRNAs}

To determine whether the growth of HCMV is inhibited in the ribozyme-expressing cells, cells were infected by HCMV at an MOI of one to five. We prepared virus stocks from the infected cultures (cells and culture medium together) at 1-d intervals through $7 \mathrm{~d}$ postinfection and determined the number of plaque forming units by measuring the viral titer on human fibroblasts. Figure 6 shows the results of the experiments. After $5 \mathrm{~d}$ postinfection, a reduction of at least 2000 -fold in viral yield was observed in cells that expressed M1-AP (Fig. 6). No significant reduction was found in those that expressed the control ribozymes C-AP or M1-TK (Fig. 6; data not shown). These results suggest that M1GSmediated targeting of viral PR and AP mRNAs effectively inhibits HCMV growth.

\section{The antiviral mechanism of M1GS RNAs targeting AP and PR mRNAs}

Although the HSV-1 capsid AP has been shown to be required for viral capsid maturation (Gao et al. 1994; Gibson et al. 1994; Matusick-Kumar et al. 1994), it has not been 


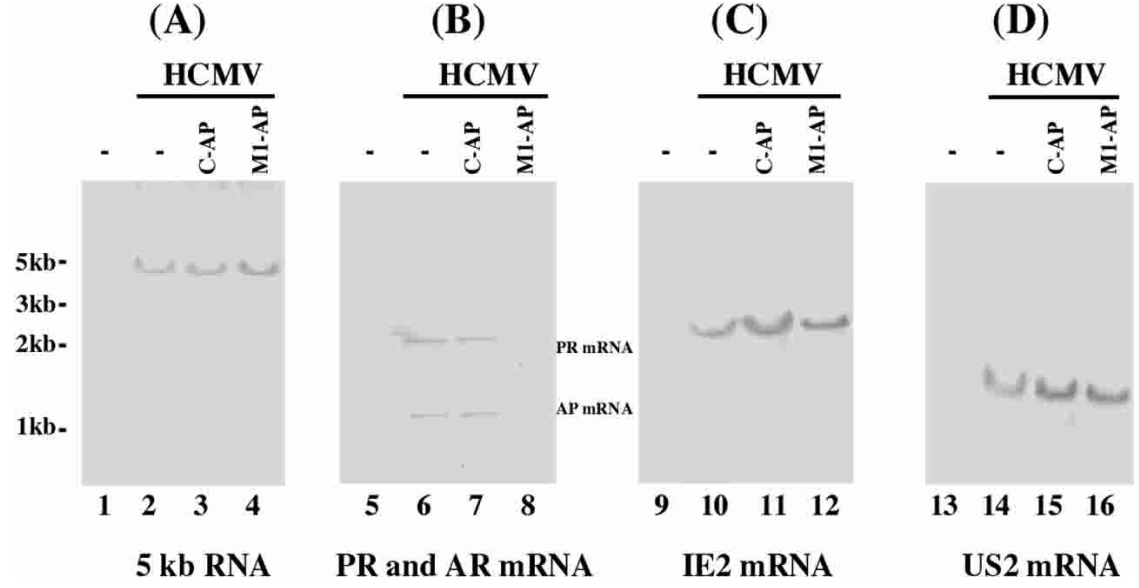

FIGURE 4. Expression levels of HCMV mRNAs as determined by Northern analysis; $5 \times 10^{5}$ cells were either mock-infected (lanes $1,5,9,13$ ) or infected with HCMV (MOI $=1$; lanes 2-4, $6-8,10-12,14-16)$ and were harvested at $8-72 \mathrm{~h}$ postinfection. Northern analyses were carried out by using RNA isolated from parental U373MG cells (-, lanes $1,2,5,6,9,10,13,14)$ and cell lines that expressed C-AP (lanes 3,7,11,15) and M1-AP (lanes 4,8,12,16). Equal amounts of each RNA sample $(30 \mu \mathrm{g})$ were separated on agarose gels that contained formaldehyde, transferred to a nitrocellulose membrane, and hybridized to a $\left[{ }^{32} \mathrm{P}\right]$-radiolabeled probe that contained the cDNA sequence of the HCMV 5-kb transcript (lanes 1-4), AP mRNA (lanes 5-8), IE2 mRNA (lanes 9-12), and US2 mRNA (lanes 13-16). The hybridized products corresponding to the 5-kb RNA, PR, AP, IE2, and US2 mRNAs were $~ 5,2.1,1.1,2.2$, and $1.5 \mathrm{~kb}$, respectively (Welch et al. 1991; Zhu et al. 1997; Mocarski and Courcelle 2001).

reported whether the HCMV AP is also essential for viral capsid assembly. Meanwhile, it is possible that the observed reduction of viral growth in the M1-AP-expressing cells is not necessarily due to specific M1GS RNA-mediated cleavage of AP and PR mRNAs but is due to other effects of the ribozyme on viral lytic replication that are unrelated to the consequence of the ribozyme cleavage or the inhibition of viral AP or PR expression, such as blocking the expression of viral IE genes.

To exclude these possibilities and further determine the

TABLE 1. Levels of inhibition of the expression of HCMV genes in the ribozyme-expressing cells, compared with the levels of inhibition in the parental U373MG cells that did not express a ribozyme (U373MG).

\begin{tabular}{llllll}
\hline & Viral gene & \multicolumn{5}{c}{ Ribozymes (\%) } \\
\cline { 3 - 6 } & class & U373MG & M1-TK & C-AP & M1-AP \\
\hline mRNA & & & & & \\
IE2 & $\alpha$ & 0 & 1 & 2 & 3 \\
US2 & $\beta$ & 0 & 3 & 2 & 1 \\
PR & $\gamma$ & 0 & 1 & 8 & $85 \pm 9$ \\
AP & $\gamma$ & 0 & 1 & 8 & $88 \pm 9$ \\
Protein & $\alpha$ & 0 & 1 & 2 & \\
IE1/IE2 & $\beta, \gamma$ & 0 & 1 & 2 & 3 \\
UL44 & $\gamma$ & 0 & 1 & 1 & 2 \\
UL83 & $\gamma$ & 0 & 0 & 3 & $80 \pm 9$ \\
PR & $\gamma$ & 0 & 0 & 3 & $82 \pm 9$ \\
AP & & & & & \\
\hline
\end{tabular}

The values of standard deviation that were $<5 \%$ are not shown. The values shown are the means from triplicate experiments. served in cells that expressed $\mathrm{M} 1-\mathrm{AP}, \mathrm{C}-\mathrm{AP}$, or (Table 1). These resuts suggest that M1-AP specificaly in(The results suggest that M1-AP specifically inhibits the expression of AP and PR and does not affect overall viral gene expression. Moreover, our results are consistent with the notion that neither AP nor PR is required for the expression of most of viral genes.

In the second set of experiments, we investigated whether viral genomic replication, as well as capsid maturation, is affected in the cells that expressed M1-AP RNA. Total DNA was isolated from HCMV-infected cell lysates. The level of intracellular viral DNA was determined by PCR detection of HCMV IE1 sequence, using the level of $\beta$-actin DNA as the internal control. Because HCMV only replicates in an episomal form and does not integrate its DNA into the host genome (Mocarski and Courcelle 2001), the amount of the intracellular viral DNA detected by the PCR assay represents the replication level of the viral genome. No significant difference in the level of viral DNA was found in cells that expressed M1-AP, C-AP, and M1$\mathrm{TK}$, suggesting that a reduction of AP and $\mathrm{PR}$ expression by the ribozyme does not affect the step of viral genome replication (Fig. 7, lanes 1-3). To examine viral capsid formation, the level of encapsidated viral DNA was assayed to determine the level of mature capsid as- 

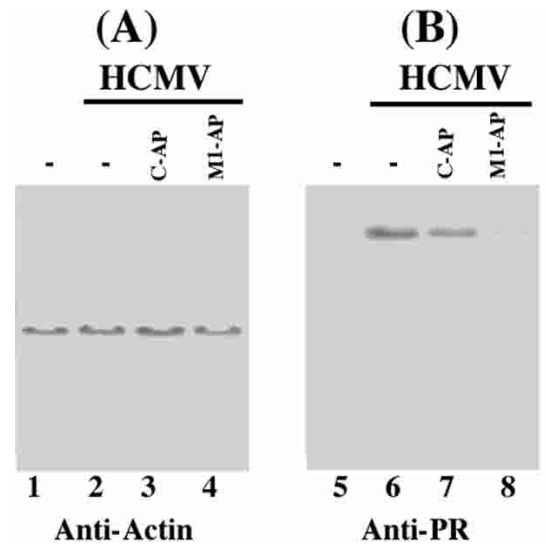

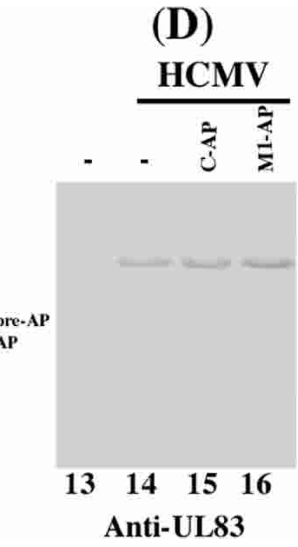

Anti-UL83

FIGURE 5. Expression of viral proteins, as detected by Western blot analysis with a chemiluminescent substrate. Total protein fractions were isolated from parental U373MG cells (-; lanes 1,2,5,6,9,10,13,14) or from cell lines that expressed C-AP (lanes 3,7,11,15) and M1-AP (lanes $4,8,12,16)$. Cells were infected with HCMV (MOI $=0.5$ to 1 ) and were harvested at 24-72 $\mathrm{h}$ postinfection. Equal amounts of protein samples $(40 \mu \mathrm{g})$ isolated from cells were separated in SDS-polyacrylamide gels. The membranes were stained with the antibodies against human actin $(A)$, HCMV PR $(B)$, HCMV AP $(C)$, and HCMV UL83 $(D)$.

sembled in the infected cells. DNA samples were isolated from HCMV-infected cell lysates that were treated with DNase I. The encapsidated viral DNAs will be resistant to DNase I digestion, whereas those that are not packaged in the capsid will be susceptible to degradation. The level of intracellular encapsidated viral DNA was determined by PCR detection of HCMV IE1 sequence. When assaying the DNA samples from cell lysates that were not treated with DNase I, we found no significant difference in the level of total intracellular (both encapsidated and uncapsidated) viral DNA in the M1GS-expressing cells (Fig. 7, lanes 1-3). When the DNase I-treated samples were assayed, however,

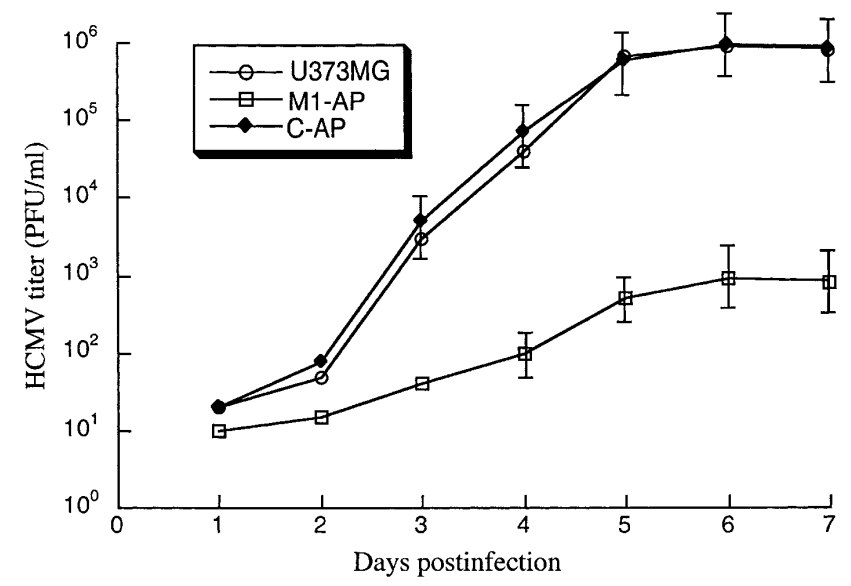

FIGURE 6. Growth of HCMV in U373MG cells and cell lines that expressed M1GS RNAs; $5 \times 10^{5}$ cells were infected with HCMV at a MOI of one to two. Virus stocks were prepared from the infected cells at 1-d intervals through 7-d postinfection, and the plaque forming units count was determined by measurement of the viral titer on human foreskin fibroblasts. The standard deviation is indicated by the error bars. These values are the means from triplicate experiments. the encapsidated DNA was hardly detected in the cells that expressed M1-AP (Fig. 7, lanes 4-6). These observations suggest that M1GS-mediated inhibition of AP and PR expression does not affect the replication of viral DNA but blocks the event(s) during or before packaging the viral genome into the capsids.

\section{DISCUSSION}

Using ribozyme to inactivate gene of choice represents an appealing approach for gene therapy, because ribozyme cleavage of an mRNA target is highly specific and is irreversible. However, for M1GS ribozyme to be successful as a therapeutic tool, the enzyme has to be highly active and the mechanism of delivery has to be extremely efficient. We have designed a M1GS RNA targeting the overlapping region of HCMV PR and AP mRNAs. The ribozyme cleaved the target mRNA efficiently in vitro and, furthermore, reduced the expression level of AP and PR by $80 \%-82 \%$ and inhibited viral growth by 2000 -fold in cells that expressed the ribozyme. Compared with levels for our controls, a reduction of $<10 \%$ in the levels of AP and PR expression and viral growth was observed in cells that expressed C-AP or M1-TK. Because M1-TK targets an unrelated mRNA, these results suggested that the observed inhibitory effect in the M1-AP-expressing cells is not due to the nonspecific effect of the ribozyme sequence. Similarly, because C-AP is catalytically inactive and contained the identical guide sequence to M1-AP, the observed reduction in viral gene expression and inhibition of viral growth in the M1-AP-expressing cells was primarily attributed to the specific targeted cleavage by the ribozyme as opposed to the antisense effect of the guide sequence.

The activity of RNase P ribozymes appears to be specific. First, cells expressing the ribozymes appear to be healthy and indistinguishable from parental cells in term of cell growth and viability for up to three months (data not shown). Second, the inhibition of viral growth and capsid maturation seems to be due to the reduction of AP and PR, because the level of DNA encapsidation and the expression of AP and PR were found to be greatly decreased in cells expressing M1-AP and not in the control cells expressing C-AP or M1-TK (Figs. 4, 5, 7; data not shown). Third, expression of the ribozymes only inhibits the expression of the AP and PR mRNAs. We found no reduction in the expression levels of other viral genes examined (e.g., IE2, US2, and UL83) in M1GS-expressing cells (Figs. 4, 5; data not shown). Finally, the replication of viral genomic DNA does not appear to be affected by the expression of M1-AP and the subsequent reduction of AP and PR expression (Fig. 


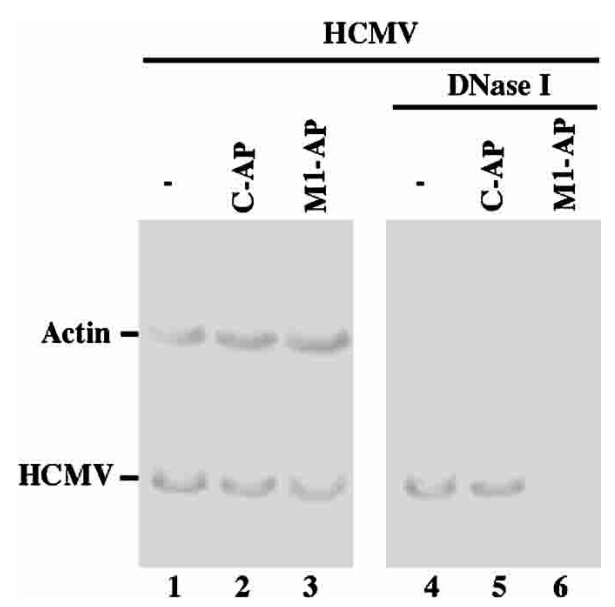

FIGURE 7. Level of total intracellular $(A)$ and encapsidated $(B)$ viral DNA as determined by semiquantative PCR. Total DNA (lanes 1-3) or DNase I-treated DNA samples (lanes 4-6) were isolated from cells that either did not express a ribozyme $(-$, lanes 1,4$)$ or expressed ribozyme C-AP (lanes 2,5) or M1-AP (lanes 3,6) and were infected with HCMV at MOI of one. We determined the levels of viral IE1 sequence by PCR with primers amplifying IE1 sequence and by using human $\beta$-actin DNA sequence as the internal controls. The radiolabeled PCR products were separated in $4 \%$ nondenaturing polyacrylamide gels and quantitated with a STORM840 PhosphorImager. The amplification by PCR was within the linear range.

7). Together, these results support the belief that AP and PR are essential for capsid maturation and do not involve in viral gene expression and genome replication.

We showed that the M1GS RNAs introduced into cultured cells were stably expressed and localized primarily in the nuclei. The expression cassette we used to produce these M1GSs is driven by the promoter for small nuclear U6 RNA. This promoter has been extensively used to express functional RNAs and ribozymes for gene targeting applications, and the transcript from this promoter is quite stable and primarily localized in the nuclei (Das et al. 1988; Yuan et al. 1992; Liu and Altman 1995; Bertrand et al. 1997; Good et al. 1997). Nuclear localization of RNase P ribozymes may play a significant role in its efficacy in cell culture. This is because the regions of the catalytic M1 RNA domain in these M1GS RNAs, which may be homologous to those of the RNA subunit of human RNase P, are believed to interact with cellular proteins, including those associated with RNase P (Liu and Altman 1995; Altman and Kirsebom 1999). This hypothesis is consistent with previous observations that several "RNA chaperone" proteins have been shown to stimulate the activity of hammerhead and group I ribozymes by facilitating either folding of the ribozyme or dissociation of the product (Tsuchihashi et al. 1993; Bertrand and Rossi 1995). Further studies on potential interactions between M1GS RNAs and cellular proteins will provide insight into how a M1GS ribozyme functions in cultured cells.

HCMV is a member of the human herpesvirus family, which includes seven other different viruses such as HSV and Epstein-Barr virus (Kieff and Rickinson 2001; Mocarski and Courcelle 2001; Roizman and Knipe 2001). All these viruses can engage in lytic replication and establish latent infections. HCMV PR and capsid APs are believed to be involved in viral capsid maturation (Mocarski and Courcelle 2001). Their homologous proteins have been found in every other herpesviruses and are highly conserved among all herpesviruses, suggesting that these proteins may serve as the ideal targets for drug development for the treatment and prevention of the infections by HCMV and other herpesviruses. Our results presented in this study indicate that M1GS RNA-mediated inhibition of the expression of HCMV AP and PR leads to a significant ( 2000-fold) reduction of viral growth, and are consistent with the notion that blocking of the expression of these two proteins should yield effective antiviral therapy. To further evaluate the antiHCMV activity of M1GS RNA, the ribozymes can be delivered into the monocyte/macrophage-lineage cells in which HCMV is believed to establish latent infection (Mocarski and Courcelle 2001). These experiments will determine whether the ribozymes can abolish viral gene expression in these cells and prevent HCMV reactivation from latent infection into lytic replication.

The M1GS-based technology represents an attractive approach for gene inactivation because it generates catalytic and irreversible cleavage of the target RNA by using M1 RNA, a highly active RNA enzyme found in nature (Frank and Pace 1998; Altman and Kirsebom 1999). These properties, as well as the simple design of the guide sequence, make M1GS an attractive and unique gene-targeting agent that can be generally used for antiviral and other in vivo applications. Indeed, our laboratory has recently showed that M1GS RNA inhibited the expression of HCMV IE1/IE2 and HSV-1 ICP4 protein and the replication of HCMV and HSV-1 (Trang et al. 2000a,b). The levels of inhibition of AP and $\mathrm{PR}$ expression observed in this study are similar to those of inhibition of the expression of HSV-1 ICP4 protein and HCMV IE1/IE2. However, the level of inhibition (2000fold) of HCMV growth by M1-AP is twofold better than that of inhibition of HSV-1 replication and at least 10-fold higher than that by the ribozyme against IE1/IE2 mRNAs (Trang et al. 2000a,b). Thus, the efficacy of the ribozyme in inhibiting viral growth might be dependent on the nature of the gene target. Our results also suggest that targeting both viral regulatory and stoichiometric functions may yield better antiviral efficacy, as AP and PR serve as regulatory and structural proteins during viral propagation, whereas IE1/ IE2 and ICP4, the viral major transcription factors (Mocarski and Courcelle 2001; Roizman and Knipe 2001), encode only the regulatory functions. Further studies on determining the best viral target for the ribozymes, as well as increasing the activity and efficacy of the M1GS ribozymes, will significantly facilitate the development of more active and effective ribozymes for both in vitro and in vivo applications. 


\section{MATERIALS AND METHODS}

\section{Ribozyme and substrate constructs}

The DNA sequence that encodes substrate ap40 was constructed by PCR using pGEM3zf(+) as a template and oligonucleotides AF25 (5'-GGAATTCTAATACGACTCACTATAG-3') and sAP2 (5'-CGGGATCCGCCGGCGACGCACCTGCCACGGTAGCACCATC TATAGTGAGTCGTATTA- $3^{\prime}$ ) as $5^{\prime}$ and $3^{\prime}$ primers, respectively. Plasmid pFL117 and pC102 contain the DNA sequence coding for M1 RNA and mutant C102 driven by the T7 RNA polymerase promoter (Kim et al. 1997; Kilani et al. 2000). Mutant ribozyme $\mathrm{C} 102$ contains several point mutations (e.g., $\left.\mathrm{A}_{347} \mathrm{C}_{348} \rightarrow \mathrm{C}_{347} \mathrm{U}_{348}, \quad \mathrm{C}_{353} \mathrm{C}_{354} \mathrm{C}_{355} \mathrm{G}_{356} \rightarrow \mathrm{G}_{353} \mathrm{G}_{354} \mathrm{~A}_{355} \mathrm{U}_{356}\right)$ at the catalytic domain (P4 helix; Kim et al. 1997). The DNA sequences that encode ribozymes M1-AP and C-AP were constructed by PCR using the DNA sequences of the M1 and C102 ribozymes as the templates and oligonucleotides AF25 and M1AP2 (5' -CCCGCTCGAGAAAAAATGGTGTGGCAGGTGCGTCGCCGT GTGGAATTGTG-3') as $5^{\prime}$ and $3^{\prime}$ primers, respectively.

\section{Cleavage and binding analysis}

M1GS RNAs and the ap40 mRNA substrate were synthesized in vitro by T7 RNA polymerase (Promega Inc.), following the manufacturer's recommendations, and further purified on $8 \%$ polyacrylamide gels containing $8 \mathrm{M}$ urea. Subsequently, the M1GS RNAs were mixed with the $\left[{ }^{32} \mathrm{P}\right]$-labeled mRNA substrate. The cleavage reactions were carried out in a volume of $10 \mu \mathrm{L}$ for 30 min at $37^{\circ} \mathrm{C}$ in buffer A $\left(50 \mathrm{mM}\right.$ Tris at $\mathrm{pH} 7.5,100 \mathrm{mM} \mathrm{NH}_{4} \mathrm{Cl}$, and $100 \mathrm{mM} \mathrm{MgCl}_{2}$; Kilani et al. 2000). Cleavage products were separated in denaturing gels and quantitated with a STORM840 phosphorimager (Molecular Dynamics). The procedures to measure the equilibrium $\mathrm{K}_{\mathrm{d}}$ of the M1GS-ap40 complexes were modified from Pyle et al (1990) and have been described previously (Trang et al. 2000b). The values of $\mathrm{K}_{\mathrm{d}}$ obtained were the average of three experiments.

\section{Viruses, cells, and antibodies}

Human primary foreskin fibroblasts (HFFs), astrocytoma U373MG cells, and PA317 cells were maintained in Dulbecco's modified Eagle's medium (DMEM) supplemented with 10\% (v/v) fetal bovine serum. The propagation of HCMV (AD169) in HFFs and U373MG cells was carried out as described previously (Trang et al. 2000b). The monoclonal antibodies c1201, c1202, c1203, and c1205, which react with HCMV proteins gB, UL44, IE1/IE2, and UL83, were purchased from Goodwin Institute for Cancer Research. The anti-rabbit polyclonal antibodies against HCMV PR and capsid AP were kindly provided by Annette Meyer of ParkeDavis Pharmaceutical Research Institute of Warner Lambert. The monoclonal antibody against human actin was purchased from Sigma.

\section{Construction of ribozyme-expressing cells}

The protocols for the construction of U373MG cells expressing different ribozymes were modified from Miller and Rosman
(1989) and have been described previously (Liu and Altman 1995). In brief, amphotropic PA317 cells were transfected with retroviral vector DNAs (LXSN-M1-AP and LXSN-C-AP) with the aid of a mammalian transfection kit purchased from GIBCO BRL. Fortyeight hours posttransfection, culture supernatants that contained retroviral vectors were collected and used to infect U373MG cells. At $48-72 \mathrm{~h}$ postinfection, cells were incubated in culture medium that contained $600 \mu \mathrm{g} / \mathrm{mL}$ neomycin. Cells were subsequently selected in the presence of neomycin for $2 \mathrm{wk}$, and neomycin-resistant cells were cloned.

For Northern analyses of the expression of the ribozymes, both nuclear and cytoplasmic RNA fractions from M1GS-expressing cells were isolated as described previously (Kawa et al. 1998). The RNA fractions were separated in a $2.5 \%$ agarose gel that contained formaldehyde, transferred to a nitrocellulose membrane, hybridized with the $\left[{ }^{32} \mathrm{P}\right]$-radiolabeled DNA probe that contained the DNA sequence coding for M1 RNA, and finally analyzed with a STORM840 phosphorimager. The radiolabeled DNA probe used to detect M1GS RNAs was synthesized from plasmid pFL117, by using a random primed labeling kit (Boehringer Manheim).

\section{Viral infection and assays for viral gene expression and growth}

T-25 flasks of cells $\left(\sim 10^{6}\right.$ cells) were either mock-infected or infected with HCMV as described previously (Trang et al. 2000b). The MOI is specified as that in the Results section. The infected cells were incubated for 8 to $72 \mathrm{~h}$, and viral mRNAs or proteins were isolated as described previously (Liu and Altman 1995). To measure the levels of viral IE transcripts, some of the cells were also treated with $100 \mu \mathrm{g} / \mathrm{mL}$ cycloheximide prior to and during infection.

The RNA fractions were separated in 1\% agarose gels that contained formaldehyde, transferred to a nitrocellulose membrane, hybridized with the $\left[{ }^{32} \mathrm{P}\right]$-radiolabeled DNA probes that contained the HCMV or human $\beta$-actin DNA sequences, and analyzed with a STORM840 Phosphorimager. The DNA probes used to detect M1GS RNAs, human $\beta$-actin mRNA, HCMV IE 5-kb RNA transcript, IE2 mRNA, US2 mRNA, and PR and AP mRNA were synthesized from plasmids pFL117, $\mathrm{p} \beta$-actin RNA, pCig27, pIE2, pCig38, and pUL80, respectively. For Western analyses, we separated the polypeptides from cell lysates on either SDS/7.5\% polyacrylamide gels or SDS/9\% polyacrylamide gels cross-linked with $N, N^{\prime \prime}$ methylenebisacrylamide. We then transferred the separated polypeptides electrically to nitrocellulose membranes. We stained the membranes by using the antibodies against HCMV proteins and human actin in the presence of a chemiluminescent substrate (Amersham Inc.), and analyzed the stained membranes with a STORM840 phosphorimager. Quantitation was performed in the linear range of RNA and protein detection.

To determine the level of the inhibition of viral growth, $5 \times 10^{5}$ cells were either mocked infected or infected with HCMV at an MOI of one to five. The infection was carried out by incubating cells with DMEM in the absence or presence of viruses for $90 \mathrm{~min}$ at $37^{\circ} \mathrm{C}$, and then with fresh DMEM for different periods of time (Trang et al. 2000b). The cells and medium were harvested at 1, 2, $3,4,5,6$, and $7 \mathrm{~d}$ postinfection, and viral stocks were prepared by adding $10 \%$ skim milk followed by sonication. The titers of the viral stocks were determined by performing plaque assays on HFFs 
(Trang et al. 2000b). The values obtained were the average from triplicate experiments.

\section{Assaying the level of viral genome replication}

The $5 \times 10^{5}$ cells grown on six-well plates were mock-infected or infected with HCMV. After a 1.5 -h incubation at $37^{\circ} \mathrm{C}$, the inoculum was removed, and the cells were further incubated and harvested at 48-96 h postinfection. Total and encapsidated (DNase I-treated) DNAs were isolated essentially as described (Gao et al. 1994; Matusick-Kumar et al. 1994) and used as the PCR DNA templates.

Viral DNA was detected by PCR amplification of the viral IE1/ IE2 sequence, using human $\beta$-actin sequence as the internal control. The $5^{\prime}$ and $3^{\prime}$ primers were CMV3 (5'-CCAAGCGGCCTC TGATAACCAAGCC- $\left.3^{\prime}\right)$ and CMV4 (5'-CAGCACCATCCTCC TCTTCCTCTGG-3'), respectively (Demmler et al. 1988), whereas those used to amplify the actin sequence were Actin5 (5'-TGAC GGGGTCACCCACACTGTGCCCATCTA-3') and Actin3 (5'CTAGAAGCATTGCGGTGGCAGATGGAGGG-3'), respectively (Daftarian et al. 1996). The PCR reaction consisted of 20 cycles with denaturation for $1 \mathrm{~min}$ at $94^{\circ} \mathrm{C}$, followed by primer annealing for $1 \mathrm{~min}$ at $47^{\circ} \mathrm{C}$ and extension for $1 \mathrm{~min}$ at $72^{\circ} \mathrm{C}$. The last cycle was again an extension for $10 \mathrm{~min}$ at $72^{\circ} \mathrm{C}$. PCR cycles and other conditions were optimized to assure that the amplification was within the linear range.

We carried out the PCR reactions in the presence of $\alpha-\left[{ }^{32} \mathrm{P}\right]-$ dCTP. The radiolabeled DNA samples were separated on polyacrylamide gels and then scanned with a STORM840 phosphorimager. We also generated a standard (dilution) curve by amplifying different dilutions of the template DNA. The plot of counts for both HCMV and $\beta$-actin versus dilutions of DNA did not reach a plateau for the saturation curve (data not shown) under the conditions described above, indicating that quantitation of viral DNA could be accomplished. Moreover, we observed that the ratio of viral DNA to $\beta$-actin remained constant with respect to each DNA dilution in the standard curve, suggesting that the assay is adequately accurate and reproducible. The PCR results were derived from three independent experiments.

\section{ACKNOWLEDGMENTS}

We thank Annette Meyer of Parke-Davis Pharmaceutical Research Institute of Warner Lambert for anti-PR and anti-AP antibodies and UL80 plasmid constructs. P.T. is a recipient of a predoctoral fellowship from American Heart Association (Western States Affiliate). K.K. is partially supported by a Block Grant Predoctoral Fellowship (UC-Berkeley). F.L. is a Pew Scholar in Biomedical Sciences, a Scholar of Leukemia and Lymphoma Society, and a recipient of an Established Investigator Award of American Heart Association. The research has been supported by grants from March of Dimes Birth Defects Foundation, American Heart Association, and National Institutes of Health.

The publication costs of this article were defrayed in part by payment of page charges. This article must therefore be hereby marked "advertisement" in accordance with 18 USC section 1734 solely to indicate this fact.

Received September 8, 2003; accepted November 7, 2003.

\section{REFERENCES}

Altman, S. and Kirsebom, L.A. 1999. Ribonuclease P. In The RNA world (eds. R.F. Gesteland et al.), pp. 351-380. Cold Spring Harbor Laboratory Press, Cold Spring Harbor, NY.

Ares, M. and Igel, A.H. 1990. Lethal and temperature-sensitive mutations and their suppressors identify an essential structural element in U2 small nuclear RNA. Genes \& Dev. 4: 2132-2145.

Bertrand, E.L. and Rossi, J.J. 1995. Facilitation of hammerhead ribozyme catalysis by the nucleocapsid protein of HIV-1 and the heterogeneous nuclear ribonucleoprotein A1. EMBO J. 13: 29042912.

Bertrand, E., Castanotto, D., Zhou, C., Carbonnelle, C., Lee, N.S., Good, P., Chatterjee, S., Grange, T., Pictet, R., Kohn, D., et al. 1997. The expression cassette determines the functional activity of ribozymes in mammalian cells by controlling their intracellular localization. RNA 3: 75-88.

Chee, M.S., Bankier, A.T., Beck, S., Bohni, R., Brown, C.M., Cerny, R., Horsnell, T., Hutchison, C.A., Kouzarides, T., and Martignetti, J.A. 1990. Analysis of the protein-coding content of the sequence of human cytomegalovirus strain AD169. Curr. Top. Microbiol. Immunol. 154: 125-169.

Cobaleda, C. and Sanchez-Garcia, I. 2000. In vivo inhibition by a site-specific catalytic RNA subunit of RNase P designed against the BCR-ABL oncogenic products: A novel approach for cancer treatment. Blood 95: 731-737.

Daftarian, P.M., Kumar, A., Kryworuchko, M., and Diaz-Mitoma, F. 1996. IL-10 production is enhanced in human T cells by IL-12 and IL- 6 and in monocytes by tumor necrosis factor- $\alpha$. J. Immunol. 157: $12-20$.

Das, G., Henning, D., Wright, D., and Reddy, R. 1988. Upstream regulatory elements are necessary and sufficient for transcription of a U6 RNA gene by RNA polymerase III. EMBO J. 7: 503-512.

Demmler, G.J., Buffone, G.J., Schimbor, C.M., and May, R.A. 1988. Detection of cytomegalovirus in urine from newborns by using polymerase chain reaction DNA amplification. J. Infect. Dis. 158: $1177-1184$.

Forster, A.C. and Altman, S. 1990. External guide sequences for an RNA enzyme. Science 249: 783-786.

Frank, D., Harris, M., and Pace, N.R. 1994. Rational design of selfcleaving pre-tRNA-ribonuclease P RNA conjugates. Biochemistry 33: $10800-10808$

Frank, D.N. and Pace, N.R. 1998. Ribonuclease P: Unity and diversity in a tRNA processing ribozyme. Ann. Rev. Biochem. 67: 153-180.

Gao, M., Matusick-Kumar, L., Hurlburt, W., DiTusa, S.F., Newcomb, W.W., Brown, J.C., McCann, P.J., Deckman, I., and Colonno, R.J. 1994. The protease of herpes simplex virus type 1 is essential for functional capsid formation and viral growth. J. Virol. 68: 37023712.

Gibson, W., Welch, A.R., and Ludford, J.M. 1994. Transient transfection assay of the herpesvirus maturational proteinase, assemblin. Methods Enzymol. 244: 399-411.

Good, P.D., Krikos, A.J., Li, S.X., Bertrand, E., Lee, N.S., Giver, L., Ellington, A., Zaia, J.A., Rossi, J.J., and Engelke, D.R. 1997. Expression of small, therapeutic RNAs in human cell nuclei. Gene Ther. 4: 45-54.

Guerrier-Takada, C., Gardiner, K., Marsh, T., Pace, N., and Altman, S. 1983. The RNA moiety of ribonuclease $\mathrm{P}$ is the catalytic subunit of the enzyme. Cell 35: 849-857.

Guo, H., Karberg, M., Long, M., Jones III, J.P., Sullenger, B., and Lambowitz, A.M. 2000. Group II introns designed to insert into therapeutically relevant DNA target sites in human cells. Science 289: 452-457.

Kawa, D., Wang, J., Yuan, Y., and Liu, F. 1998. Inhibition of viral gene expression by human ribonuclease P. RNA 4: 1397-1406.

Kieff, E. and Rickinson, A.B. 2001. Epstein-Barr viruses and its replication. In Fields virology, Vol. 2 (eds. D.M. Knipe and P.M. Howley), pp. 2511-2573. Lippincott-Williams \& Wilkins, Philadelphia, PA. 
Kilani, A.F., Trang, P., Jo, S., Hsu, A., Kim, J., Nepomuceno, E., Liou, K., and Liu, F. 2000. RNase P ribozymes selected in vitro to cleave a viral mRNA effectively inhibit its expression in cell culture. $J$. Biol. Chem. 275: 10611-10622.

Kim, J.J., Kilani, A.F., Zhan, X., Altman, S., and Liu, F. 1997. The protein cofactor allows the sequence of an RNase P ribozyme to diversify by maintaining the catalytically active structure of the enzyme. RNA 3: 613-623.

Lan, N., Howrey, R.P., Lee, S.W., Smith, C.A., and Sullenger, B.A. 1998. Ribozyme-mediated repair of sickle $\beta$-globin mRNAs in erythrocyte precursors. Science 280: 1593-1596.

Liu, F. and Altman, S. 1995. Inhibition of viral gene expression by the catalytic RNA subunit of RNase P from Escherichia coli. Genes \& Dev. 9: 471-480.

1996. Requirements for cleavage by a modified RNase P of a small model substrate. Nucleic Acids Res. 24: 2690-2696.

Liu, F. and Roizman, B. 1991. The herpes simplex virus 1 gene encoding a protease also contains within its coding domain the gene encoding the more abundant substrate. J. Virol. 65: 5149-5156.

Matusick-Kumar, L., Hurlburt, W., Weinheimer, S.P., Newcomb, W.W., Brown, J.C., and Gao, M. 1994. Phenotype of the herpes simplex virus type 1 protease substrate ICP35 mutant virus. J. Virol. 68: 5384-5394.

Miller, A.D. and Rosman, G.J. 1989. Improved retroviral vectors for gene transfer and expression. BioTechniques 7: 980-990.

Mocarski, E.S. and Courcelle, C.T. 2001. Cytomegaloviruses and their replication. In Fields virology (eds. D.M. Knipe and P.M. Howley), pp. 2629-2673. Lippincott-Williams \& Wilkins, Philadelphia, PA.

Moore, P.S. and Chang, Y. 2001. Kaposi's sarcoma-associated herpesvirus. In Fields virology (eds. D.M. Knipe and P.M. Howley), pp. 2803-2834. Lippincott-Williams \& Wilkins, Philadelphia, PA.

Pass, R.F. 2001, Cytomegalovirus. In Fields virology (eds. D.M. Knipe and P.M. Howley), pp. 2675-2706. Lippincott-Williams \& Wilkins, Philadelphia, PA.

Preston, V.G., Coates, J.A., and Rixon, F.J. 1983. Identification and characterization of a herpes simplex virus gene product required for encapsidation of virus DNA. J.Virol. 45: 1056-1064.

Pyle, A.M., McSwiggen, J.A., and Cech, T.R. 1990. Direct measurement of oligonucleotide substrate binding to wild-type and mutant ribozymes from Tetrahymena. Proc. Natl. Acad. Sci. 87: 8187-8191.

Roizman, B. and Knipe, D.M. 2001. Herpes simplex viruses and their replication. In Fields virology (eds. D.M. Knipe and P.M. Howley), pp. 2399-2460. Lippincott-Williams \& Wilkins, Philadelphia, PA.

Rossi, J.J. 1999. Ribozymes, genomics and therapeutics. Chem. Biol. 6: R33-R37.

2000. Ribozyme therapy for HIV infection. Adv. Drug. Del. Rev. 44: 71-78.

Sarver, N., Cantin, E.M., Chang, P.S., Zaia, J.A., Ladne, P.A., Stephens, D.A., and Rossi, J.J. 1990. Ribozymes as potential anti-HIV-1 therapeutic agents. Science 247: 1222-1225.

Trang, P., Kilani, A.F., Kim, J., and Liu, F. 2000a. A ribozyme derived from the catalytic subunit of RNase P from Escherichia coli is highly effective in inhibiting replication of herpes simplex virus 1. J. Mol. Biol. 301: 817-826.

Trang, P., Lee, M., Nepomuceno, E., Kim, J., Zhu, H., and Liu, F. 2000b. Effective inhibition of human cytomegalovirus gene expression and replication by a ribozyme derived from the catalytic RNA subunit of RNase P from Escherichia coli. Proc. Natl. Acad. Sci. 97: 5812-5817.

Tsuchihashi, Z., Khosla, M., and Herschlag, D. 1993. Protein enhancement of hammerhead ribozyme catalysis. Science 262: 99-102.

Welch, A.R., Woods, A.S., McNally, L.M., Cotter, R.J., and Gibson, W. 1991. A herpesvirus maturational proteinase, assemblin: Identification of its gene, putative active site domain, and cleavage site. Proc. Natl. Acad. Sci. 88: 10792-10796.

Wong-Staal, F., Poeschla, E.M., and Looney, D.J. 1998. A controlled, phase 1 clinical trial to evaluate the safety and effects in HIV-1infected humans of autologous lymphocytes transduced with a ribozyme that cleaves HIV-1 RNA. Hum. Gene Ther. 9: 2407-2425.

Yu, M., Ojwang, J., Yamada, O., Hampel, A., Rapapport, J., Looney, D., and Wong-Staal, F. 1993. A hairpin ribozyme inhibits expression of diverse strains of human immunodeficiency virus type 1. Proc. Natl. Acad. Sci. 90: 6340-6344.

Yuan, Y., Hwang, E., and Altman, S. 1992. Targeted cleavage of mRNA by human RNase P. Proc. Natl. Acad. Sci. 89: 8006-8010.

Zaug, A.J. and Cech, T.R. 1995. Analysis of the structure of Tetrahymena nuclear RNAs in vivo: Telomerase RNA, the self-splicing rRNA intron, and U2 snRNA. RNA 1: 363-374.

Zhu, H., Cong, J.P., and Shenk, T. 1997. Use of differential display analysis to assess the effect of human cytomegalovirus infection on the accumulation of cellular RNAs: Induction of interferon-responsive RNAs. Proc. Natl. Acad. Sci. 94: 13985-13990. 

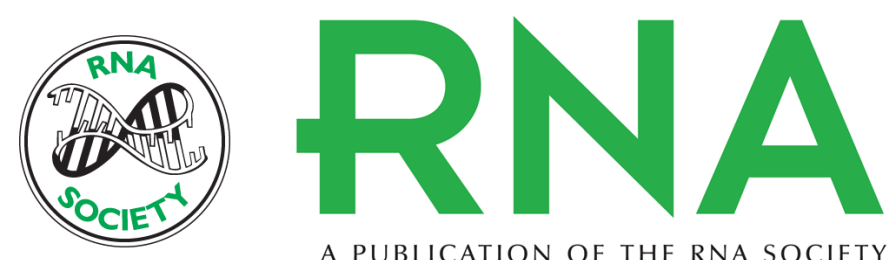

A PUBLICATION OF THE RNA SOCIETY

\section{Intracellular expression of engineered RNase P ribozymes effectively blocks gene expression and replication of human cytomegalovirus}

KIHOON KIM, SEAN UMAMOTO, PHONG TRANG, et al.

RNA 2004 10: 438-447

References This article cites 39 articles, 24 of which can be accessed free at:

http://rnajournal.cshlp.org/content/10/3/438.full.html\#ref-list-1

License

Email Alerting Receive free email alerts when new articles cite this article - sign up in the box at the Service top right corner of the article or click here. 